\title{
Complex Compound Odontoma Associated with an Unerupted Tooth: A Rare Entity
}

\author{
Shilpa Shetty', Aishwarya Kamble ${ }^{2}$, Farhin Katge ${ }^{3^{*}}$ and Komal Nanavati ${ }^{4}$ \\ 'Reader, Department of Pediatric and Preventive Dentistry, Terna Dental College, Sector 22, Plot 12, \\ Phase II, Nerul (west), Navi Mumbai - 400706, India \\ 2Postgraduate Student, Department of Pediatric and Preventive Dentistry, Terna Dental College, \\ Sector 22, Plot 12, Phase II, Nerul (west), Navi Mumbai - 400706; India \\ ${ }^{3}$ Head of Department, Department of Pediatric and Preventive Dentistry, Terna Dental College, \\ Sector 22, Plot 12, Phase II, Nerul (west), Navi Mumbai - 400706, India; pedotdc@gmail.com \\ ${ }^{4}$ Postgraduate Student, Department of Pediatric and Preventive Dentistry, Terna Dental College, \\ Sector 22, Plot 12, Phase II, Nerul (west), Navi Mumbai - 400706, India
}

\section{Abstract}

Odontomas are odontogenic benign tumours composed of enamel, dentine, cementum, and pulp like tissue which are generally discovered during diagnostic radiography and are often asymptomatic. According to World Health Organisation (WHO), odontoma are classified as compound and complex odontomas. Compound or complex odontoma are commonly reported in the oral cavity but very rarely an odontoma may show features of both. This paper reports a rare case of an odontoma with both compound and complex features, occurring in the upper front region of the jaw associated with impaction of right permanent maxillary central incisor.

Keywords: Complex Compound Odontoma, Odontogenic Tumour, Unerupted

\section{Introduction}

Odontomas are developmental anomalies resulted from growth of completely differentiated epithelial and mesenchymal cells which give rise to ameloblasts and odontoblasts. ${ }^{[1]}$ The term odontoma was first coined by Broca in 1866. He defined it as "tumors formed by the overgrowth of transitory or complete dental tissues". ${ }^{2]}$

According to WHO (2005), odontoma is an odontogenic benign tumor of young age which are tumor-like formations (hamartomas of dental tissues) or developmental anomalies, rather than true odontogenic neoplasms. ${ }^{[3]}$ Most of the odontomas are asymptomatic in nature, but signs and symptoms may occur occasionally. They may be associated to an unerupted or impacted teeth or show evidence of infection. ${ }^{[4]}$
Waldron AC (2002) defined odontomas as "Non aggressive, hamartomatous developmental malformations or lesions of odontogenic origin appearing as small, solitary or multiple radiopaque lesions found on routine radiographic examinations." ${ }^{[5]}$ Odontomas are the most common type of odontogenic tumors comprising $22 \%$ of all the odontogenic tumors of the jaws. ${ }^{[6]}$

Odontomas have been associated with: ${ }^{[7]}$

- Trauma during primary dentition.

- Inflammatory and infectious processes.

- Hereditary anomalies (Gardner syndrome, Hermann's syndrome).

- Odontoblastic hyperactivity.

- Alterations in the genetic components responsible for controlling dental development. 
According to Hitchin, odontomas are inherited through a postnatal gene mutation. There is a tendency in humans for the lamina to disintegrate into clumps of cells between the tooth germs. One of the etiologic factor in the formation of complex or compound odontomas instead of a tooth may be the persistence of a portion of dental lamina. Another important factor in aetiology is a mutation of epithelial cells of the tooth germ or persistent lamina which may itself induce the odontogenic epithelium to go through various stages of tooth formation. The persistent dental lamina may still continue to stimulate mesenchymal differentiation which is necessary for hard tissue formation. This results in formation of functional ameloblasts and odontoblasts eventually forming a composite odontoma. ${ }^{[8]}$

Gabell, James and Payne (1914) classified odontoma based on their developmental origin as ${ }^{[9,10]}$

- Epithelial.

- Composite (epithelial and mesodermal).

- Connective tissue.

According to a classification by Thoma and Goldman $(1946)^{[11]}$

- Geminated composite odontomas: Two or more, more or-less well-developed teeth fused together.

- Compound composite odontomas: Made up of more-or less rudimentary teeth.

- Complex composite odontomas: Calcified structure, which bears no great resemblance to the normal anatomical arrangement of dental tissues.

- Dilated odontomas: The crown or root part of tooth shows marked enlargement.

- Cystic odontomas: An odontome that is normally encapsulated by fibrous connective tissue in a cyst or in the wall of a cyst.

WHO classified odontomas into three groups ${ }^{[2]}$

- Complex odontoma: When the calcified dental tissues are simply arranged in an irregular mass bearing no morphologic similarity to rudimentary teeth.

- Compound odontoma: Composed of all odontogenic tissues in an orderly pattern that result in many teeth like structures, but without morphologic resemblance to normal teeth.

- Ameloblastic fibro-odontoma: Consists of varying amounts of calcified dental tissue and dental papilla-like tissue, the later component resembling an ameloblastic fibroma. The ameloblastic fibro-odontome is considered as an immature precursor of complex odontome.

The aim of this case report is to describe an interesting case of a mixed compound and complex odontoma localized in the premaxilla area associated with an impacted permanent maxillary incisor.

\section{Case Report}

A 12-year-old male patient reported to the Department of pediatric and Preventive dentistry with chief complaint of delayed eruption of teeth in upper front region of the jaw. Medical and family history of the patient was insignificant. Intraoral examination showed a missing right permanent maxillary central incisor and presence of over retained deciduous maxillary lateral incisor. There was a presence of a slight bulge in the region of unerupted tooth (Figure 1 and 2). The swelling was oval, measuring approximately $2 \times 3 \mathrm{cms}$ seen in the region of 11 and 12 . Overlying mucosa appeared normal. On palpation, the swelling was firm, non- compressible and non-tender in nature.

Radiographic examination by an Intra Oral Periapical radiograph (IOPA) revealed the presence of dense, calcified, amorphous, irregularly shaped radio-opaque mass of varying density measuring approximately $1 \mathrm{~cm}$ in diameter (Figure 3). This radio opacity was surrounded by a radiolucency having well defined borders. An Orthopantomograph (OPG) revealed the presence of tooth like structure and impaction with permanent right

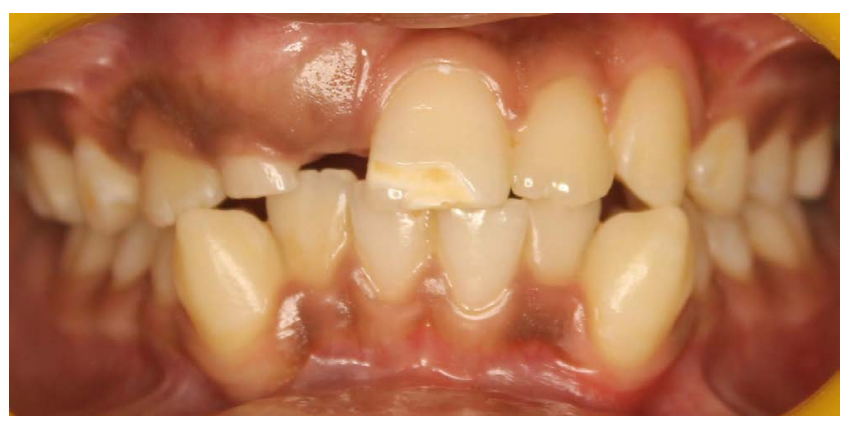

Figure 1. Intra oral photograph (frontal). 


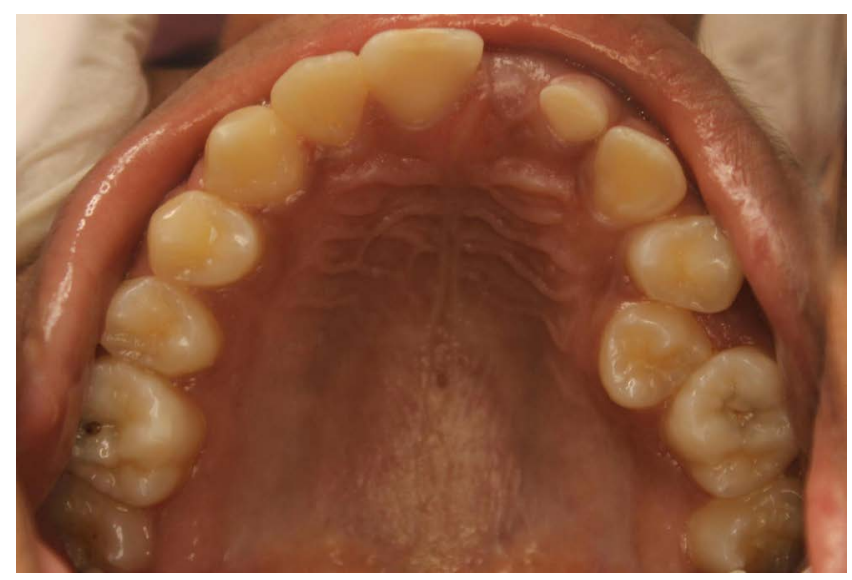

Figure 2. Intra oral photograph (occlusal).

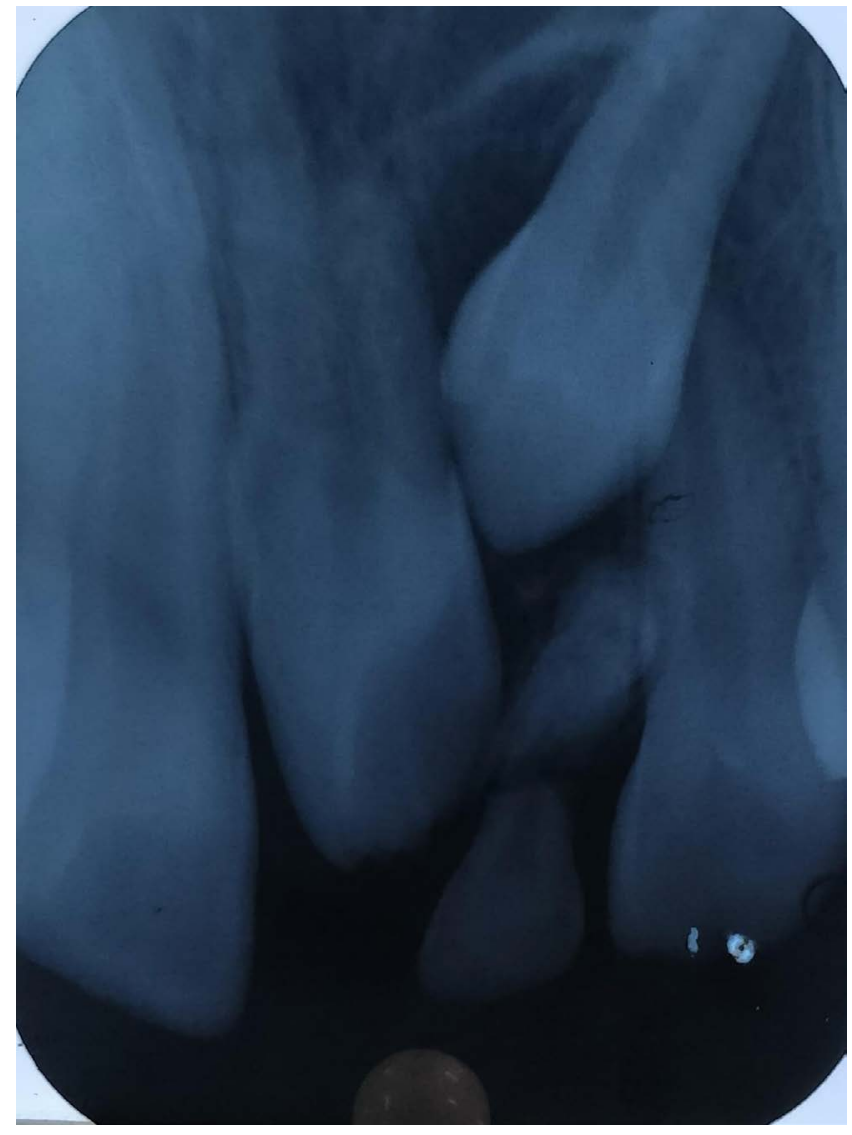

Figure 3. Intra oral periapical radiograph.

maxillary central incisor and canine could be appreciated. The OPG also detected transposition with permanent right maxillary lateral incisor and canine (Figure 4). A provisional diagnosis of odontoma was made, on the basis of the clinical and radiographic examination.

After achieving adequate local anaesthesia (2\% lignocaine with 1:1,00,000 adrenaline), mucosa was

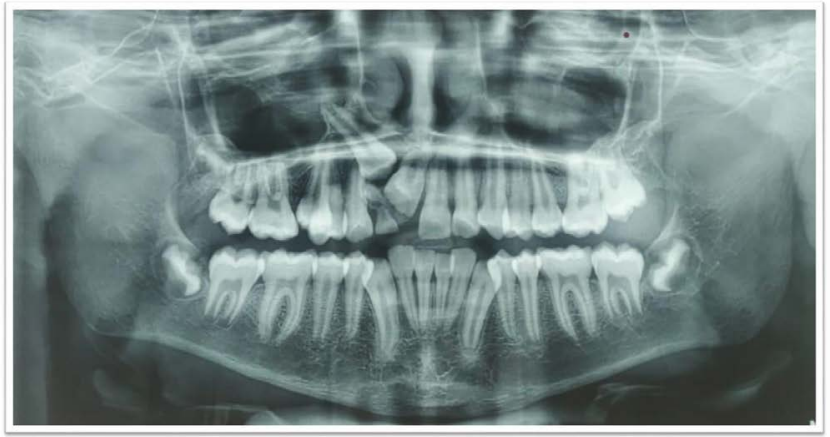

Figure 4. Orthopantomograph.

reflected on the buccal and palatal aspect of the deciduous maxillary lateral incisor using a periosteal elevator (Figure 5 and 6). The over retained deciduous tooth was extracted using a primary incisor forcep (Figure 7). The calcified tooth like structure which was present below the apex of the deciduous tooth was visualised by further reflection of the mucosa using the periosteal elevator. It was removed by elevation using a periosteal elevator without disturbing the underlying unerupted permanent canine. The specimen was then sent to the Department of Oral Pathology and Microbiology for histopathological analysis (Figure 8).

On histopathological examination, the sections revealed components of enamel organ (Figure 9). Calcified structures like enamel, dentin, and cementum which were intermingled with pulp-like tissues were present in few areas. The calcified structures were arranged regularly in few areas, whereas, in few areas it was haphazardly arranged. Large mature tubular dentin was apparently seen enclosed in clefts or hollow structures which contained immature enamel or enamel matrix. Small amount of supporting fibrous connective tissue

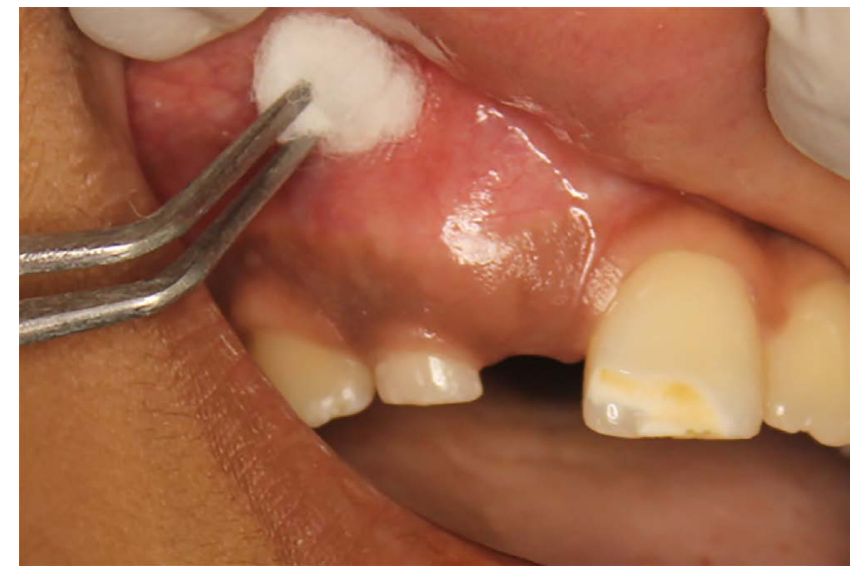

Figure 5. Application of local anaesthetic agent 2\% lignocaine. 


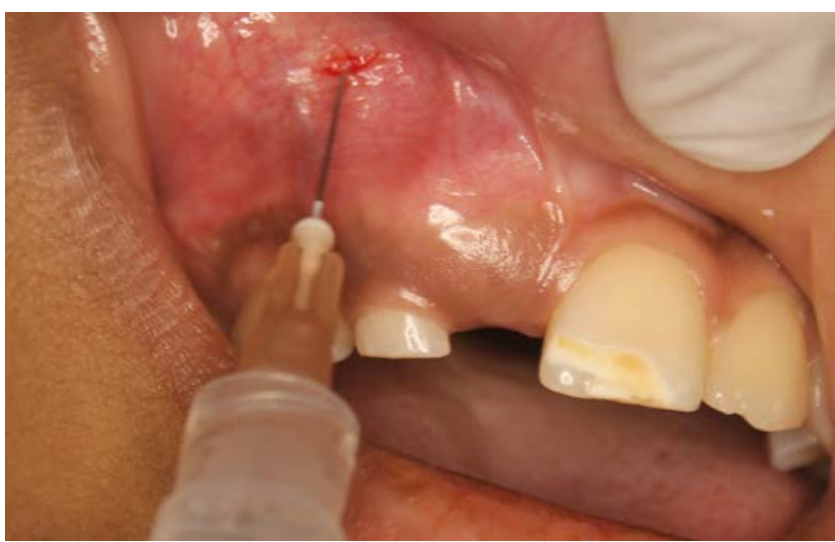

Figure 6. Buccal infiltration administration with $2 \%$ lignocaine 1:80,000 adrenaline solution.

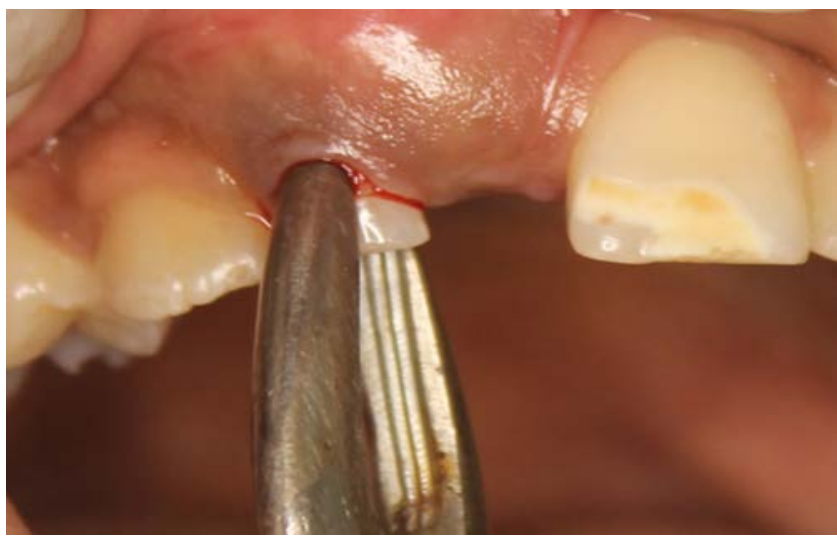

Figure 7. Extraction of 52.

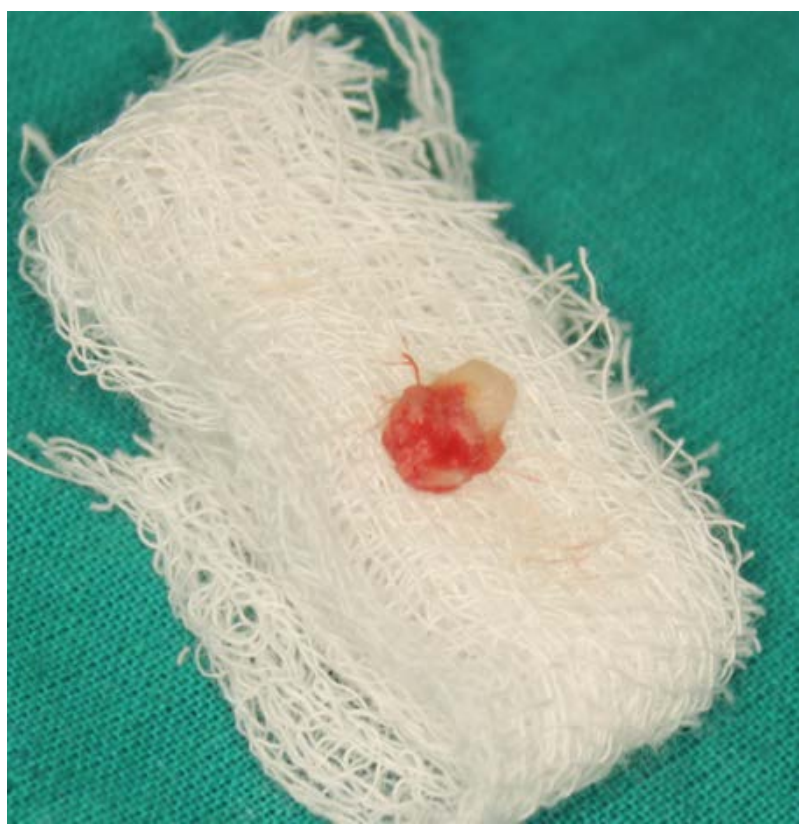

Figure 8. Extracted odontome.

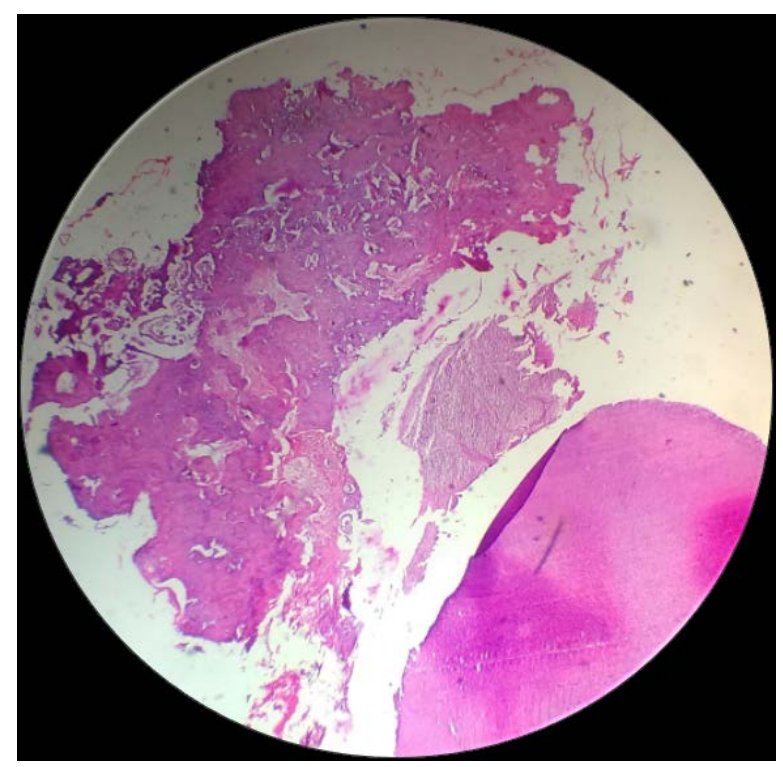

Figure 9. Under low magnification.

stroma representing fibrous capsule was also present. Few odontogenic islands were seen in a fibro-vascular connective tissue (Figure 10, 11 and 12). Based on the appearance of the gross specimen and the histopathological examination a final diagnosis of compound complex odontoma was made, which is a rare entity.

The patient was recalled after a week which showed uneventful healing of the extraction site. The patient was later referred to the Department of Orthodontics for comprehensive orthodontic treatment of the impacted teeth.

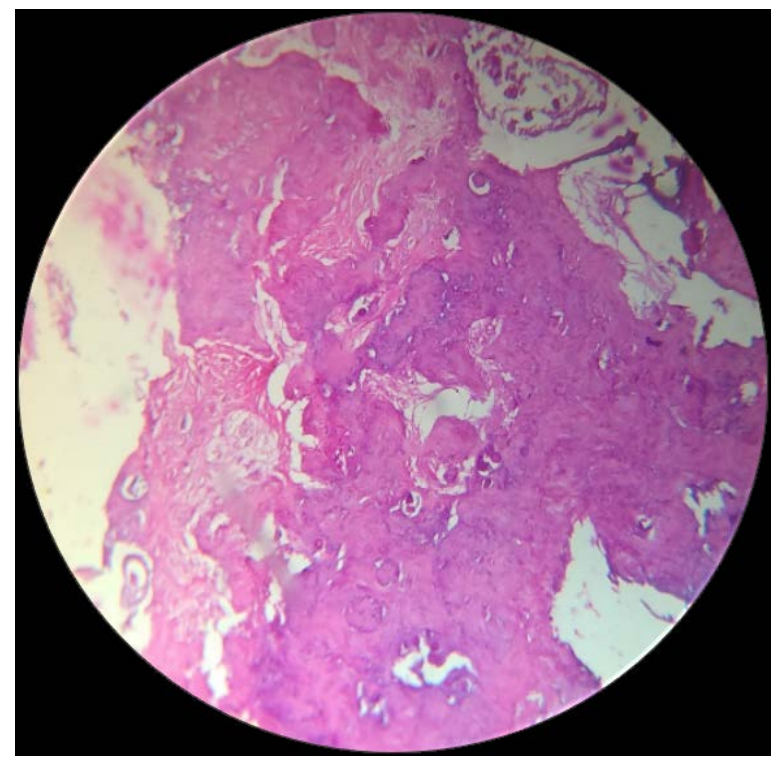

Figure 10. Disorganized masses of osteodentine enclosing clefts of enamel matrix. 


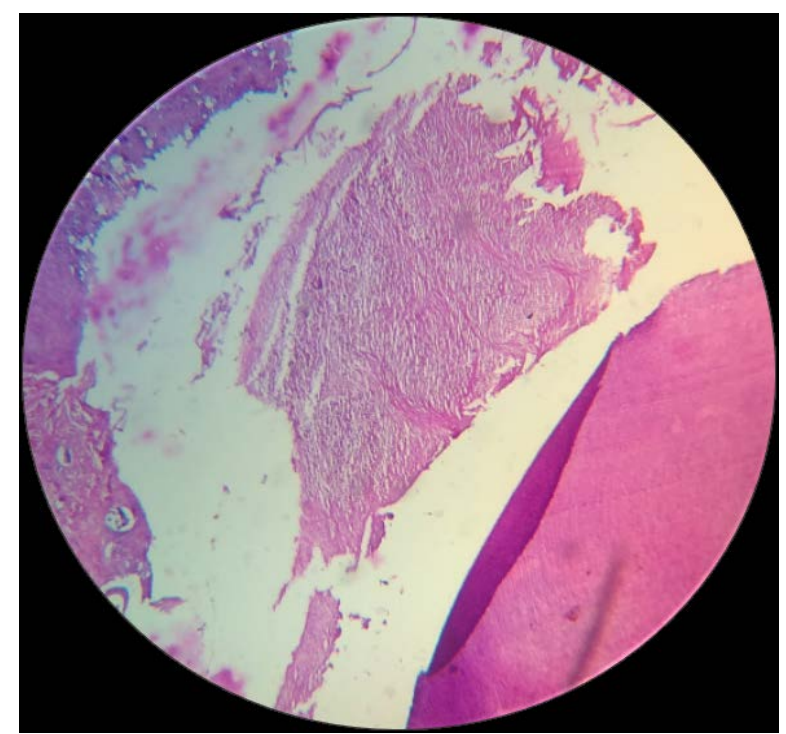

Figure 11. Haphazardly placed dentinal tubules.

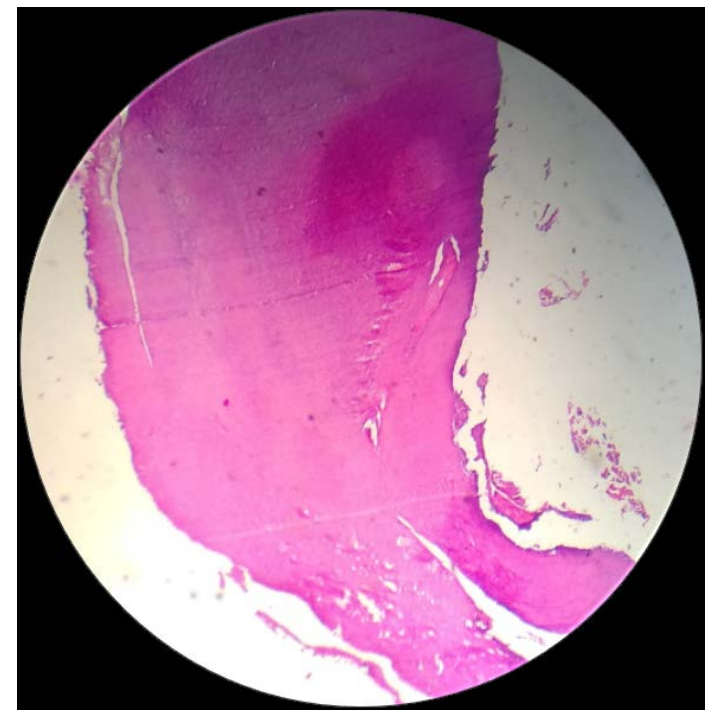

Figure 12. Section showing enamel and dentin-like structure with pulp tissue.

\section{Discussion}

The incidence of odontomas in oral cavity is in the range of $9 \%$ to $37 \%$. Compound composite odontomas are most commonly seen in anterior segment $(61 \%)$, whereas Complex composite odontomas are seen in the posterior segment (34\%). ${ }^{[4]}$ Complex (68\%) and compound (62\%) odontomas are found on the right side of the jaw more frequently than on the left. Incisor cuspid region of the upper jaw are the most frequent site for compound composite odontoma, whereas molar and premolar region of the mandible are common sites for complex

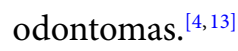

Keskin et al., found only five reports (4 case report and 1 retrospective study) about complex-compound odontoma in the literature. ${ }^{[14]}$ Thus, the present case is unique as it has combined features of both compound and complex odontoma. In a review of 160 cases of odontomas by Tekkesin et al., of all the investigated cases, 99 were complex, 57 were compound, and only four were mixed odontomas. This further confirms the rare occurrence of the mixed odontome that is the compound-complex odontoma. $^{[15]}$

Odontomas generally have a non-aggressive nature and tend to grow slowly. The most commonly observed symptoms associated with odontoma are pain, swelling, malocclusion and pathological anomalies in the adjacent teeth. $^{[16]}$ Literature shows that $37-77 \%$ of eruption disorders are seen with odontomas. ${ }^{[17]}$ The findings in the present case report are consistent with literature, as the odontoma caused impaction of the permanent incisor.

In most of the cases, odontomas are impacted within the jaw bones and rarely show eruption in the oral cavity. ${ }^{[18]}$ Although odontomas are usually intraosseous, signs like expansion of the bone are not commonly noticed. In some cases, they can be examined visually, and this phenomenon has extremely rare occurrence in the cases of compound odontomas. In the current case report, detectable expansion of the bone surrounding complexcompound odontoma could be well appreciated on clinical examination. However, the exact aetiology is still debatable. It is considered that the expansion may be due to the eruptive forces arising from impacted teeth. ${ }^{[19]}$

According to Kaban, odontomas can be enucleated with ease and adjacent teeth are rarely traumatized during surgical excision because they are usually separated by a septum of bone. ${ }^{[20]}$ Although odontoma has a limited growth potential, it should be removed as it contains various tooth formulations which can predispose to cystic change. ${ }^{[21]}$

\section{Conclusion}

Early diagnosis of odontomas will help us achieve a simpler and less expensive treatment option. It will also prevent displacement or devitalisation of adjacent vital tooth structures and ensure a better prognosis. ${ }^{[22]}$ 
All paediatric patients who report with clinical signs of missing tooth, delayed eruption or tooth displacement should be subjected to a detailed visual and radiographic examination for facilitating early diagnosis.

\section{References}

1. Neville BW, Damm DD, Allen CM, Bouquot JE. Oral and maxillofacial pathology. Philadelphia: Saunders; 1995. p. 531-33.

2. Cohen DM, Bhattacharyya I. Ameloblastic fibroma, ameloblastic fibroodontoma, and odontoma. Oral Maxillofac Surg Clin North Am. 2004; 16:375-384. https:// doi.org/10.1016/j.coms.2004.03.005 PMid:18088738

3. Kramar IRH, Pindporg JJ, Shear M.. Histological typing of odontogenic tumours, World Health Organization. International Histological Classification of Tumours. 2nd ed. Berlin, Germany: Springer; 1992. https://doi. org/10.1007/978-3-662-02858-2_2

4. Shafer, Hine, Levy. A Text Book of Oral Pathology, 4th ed. W.B. Saunders and Co; 1993. p. 308-12.

5. Waldron AC. Odontogenic cysts and tumours. Oral and Maxillofacial Pathology. Neville BW. 2nd ed. Philadelphia, Pa, USA: WB Saunders; 2002. P. 631-32.

6. Cuesta SA, Albiol JG, Aytes LB, Escoda CG. Review of 61 cases of odontoma. Presentation of an erupted complex odontoma. Medicina Oral. 2003; 8(5):366-73.

7. Piattelli A, Perfetti G, Carrano A. Complex odontome as a periapical and interradicular radioopacity in a primary molar. J Endod. 1996; 22:561-3 https://doi.org/10.1016/ S0099-2399(96)80019-7

8. Hitchin AD. The aetiology of the calcified composite odontomes. Brit Dent J. 1971; 130:475-82. https://doi. org/10.1038/sj.bdj.4802682 PMid:5281128

9. Budnick SD. Compound and complex odontomas. Oral Surgery Oral Medicine and Oral Pathology. 1976; 42(4):50106. https://doi.org/10.1016/0030-4220(76)90297-8

10. Singh S, Singh M, Singh I, Khandelwal D. Compound compositeodontomeassociated withanunerupteddeciduous incisor- A rarity. J Indian Soc Pedod Prev Dent. 2005; 23:14650. https://doi.org/10.4103/0970-4388.16889 PMid:1622 4136
11. Thoma KM, Goldman HM. Oral pathology. 5th ed. St Louis: The CV Mosby Company; 1960. p. 1221-2.

12. Kramer IR, Pindborg JJ, Shear M. Histological typing of odontogenic tumour. WHO. International Histological Classification of Tumours. 2nd ed. Berlin Springer; 1992. p. 16-21. https://doi.org/10.1007/978-3-662-02858-2

13. Pindborg JJ, Hjortiy-Hansen E. Atlas of diseases of the Jaws. Copenhagen, Denmark: Munksgaard; 1974.

14. Keskin M. Complex-compound odontoma: A rare clinical presentation. Odovtos-Int J Dent Sci. 2019: 39-44. https:// doi.org/10.15517/ijds.v0i0.33920

15. Soluk Tekkesin M, Pehlivan S, Olgac V, Aksakallı N, Alatli C. Clinical and histopathological investigation of odontomas: Review of the literature and presentation of 160 cases. J Oral Maxillofac Surg. 2012; 70:1358-61. https:// doi.org/10.1016/j.joms.2011.05.024 PMid:21840103

16. Verma S, Arul AS, Arul AS, Chitra S. Erupted complex odontoma of the posterior maxilla: A rarity. J Nat Sci Biol Med.2015;6(Suppl 1):S167-9.https://doi.org/10.4103/09769668.166130 PMid:26604611 PMCid:PMC4630755

17. Ohtawa Y, Ichinohe S, Kimura E, Hashimoto S. Erupted complex odontoma delayed eruption of permanent molar. Bull Tokyo Dent Coll. 2013; 54(4):251-7. https://doi. org/10.2209/tdcpublication.54.251 PMid:24521551

18. Serra-Serra G, Berini-Aytes L, Gay-Escoda C. Erupted odontomas: A report of three cases and review of the literature. Med Oral Patol Oral Cir Bucal. 2009; 14 (6):E299-303.

19. Hanemann JA, Oliveira DT, Garcia NG, Santos MR, Pereira AA. Peripheral compound odontoma erupting in the gingiva. Head Face Med. 2013; 9:15. https://doi. org/10.1186/1746-160X-9-15 PMid:23758697 PMCid:PMC 3684544

20. Kaban LB., Troulis MJ. Dentoalveolar surgery. Pediatric Oral and Maxillofacial Surgery. Philadelphia, PA, USA: WB Saunders; 2004. p. 140.

21. Kaban LB. Pediatric oral and maxillofacial surgery. Philadelphia, PA, USA: Saunders; 1990.

22. Satish V, Prabhadevi MC, Sharma R. Odontome: a brief overview. Int J Clin Pediatr Dent. 2011; 4(3):177-85. https:// doi.org/10.5005/jp-journals-10005-1106 PMid:27678223 PMCid:PMC5034075
How to cite this article: Shetty S., Kamble A., Katge F. and Nanavati K. Complex Compound Odontoma Associated with an Unerupted Tooth: A Rare Entity. Int. J. Med. Dent. Sci. 2020; 9(1): 1868-1873. 